\title{
Coalgebraic Predicate Logic: Equipollence Results and Proof Theory
}

\author{
Tadeusz Litak ${ }^{1 \star}$, Dirk Pattinson ${ }^{2}$, and Katsuhiko Sano ${ }^{3}$ \\ 1 FAU Erlangen-Nürnberg \\ 2 Imperial College London \\ 3 Japan Advanced Institute of Science and Technology
}

\begin{abstract}
The recently introduced Coalgebraic Predicate Logic (CPL) provides a general first-order syntax together with extra modal-like operators that are interpreted in a coalgebraic setting. The universality of the coalgebraic approach allows us to instantiate the framework to a wide variety of situations, including probabilistic logic, coalition logic or the logic of neighbourhood frames. The last case generalises a logical setup proposed by C.C. Chang in early 1970's. We provide further evidence of the naturality of this framework. We identify syntactically the fragments of CPL corresponding to extended modal formalisms and show that the full CPL is equipollent with coalgebraic hybrid logic with the downarrow binder and the universal modality. Furthermore, we initiate the study of structural proof theory for CPL by providing a sequent calculus and a cutelimination result.
\end{abstract}

\section{Introduction}

Coalgebras over sets provide an universal framework for state-based systems, such as (labelled or unlabelled) transition systems, multigraphs, conditional frames, game frames or (monotone and general) neighbourhood frames. They provide a natural semantics for a wide range of modal logics, ranging from conditional and probabilistic to coalition logic. The development of a full-blown coalgebraic model and correspondence theory is hindered by the lack of a formalism that allows both direct reference to individual states and supports universal quantification and binding: a coalgebraic counterpart of first-order (and higher-order) predicate logic. The framework of coalgebraic predicate logic (CPL) was introduced recently in [9] in a joint paper with Lutz Schröder, where we have provided a complete Hilbert axiomatisation, a modal correspondence theorem and some basic model-theoretic constructions. The present paper is intended as a companion to op.cit. presenting more evidence that coalgebraic predicate logic is a natural extension of both (coalgebraic) modal logic and first-order logic.

As explained in op.cit., our approach can be traced back to an unjustly forgotten paper [7] by C. C. Chang. The original motivation was to simplify model theory for what

\footnotetext{
* The first author gratefully acknowledges the support of the EPSRC grant EP/G041296/1 while employed at the University of Leicester. Thanks are also due to the anonymous referee for careful reading and many insightful comments and to Guram Bezhanishvili for his patience during the editing process.
} 
Montague called pragmatics and replace Montague's many-sorted setting by one without sorts. In contemporary terminology, Chang's paper deals with model and correspondence theory for neighbourhood frames: coalgebras for double contravariant powerset functor (see [8] for a coalgebraic treatment in a two-sorted setting). His constructions and results include both suitable notions of (elementary) submodel/extension, elementary chain of models and ultraproduct and suitable variants of Tarski-Vaught, downward and upward Löwenheim-Skolem and compactness theorems. Curiously, Chang did not prove any completeness results. Apart from these technical developments, advantages of [7] include its lucid, intuitive motivation and examples. But the biggest interest lies in the syntax itself, with only one sort of variables for elements of the state space and no need for explicit quantification over neighbourhood or successors. Apart from a number of papers (e.g., Sgro [16]) on interior operator logic in topology, we are not aware of any work in a similar setup.

Our CPL (Coalgebraic Predicate Logic) is based on a notational variant of Chang's syntax. The interpretation of CPL in coalgebras for arbitrary Set-functors is parametric in the notion of predicate lifting; if $\Lambda$ is a supply of modal operators, then the supply of functors and predicate liftings interpreting modalities in $\Lambda$ is called $\Lambda$-structure. In [9], the authors together with Lutz Schröder have proved completeness results for two classes of $\Lambda$-structures-the first one (S1SC) generalizing neighbourhood frames, another one ( $k$-bounded) generalizing Kripke structures. Furthermore, it was shown that there are limitations to the possible scope of more general completeness results. We also proved an analogue of the Van Benthem-Rosen theorem, characterizing coalgebraic modal logic (CML) as the behavioural-invariant fragment of CPL. Finally, we provided foundations of model theory for CPL, significantly generalizing the scope of Chang's model-theoretic results discussed above.

This paper is intended as a companion paper to [9]. In the first part, we generalize the results of [4] characterizing the correspondence between predicate logic and extended

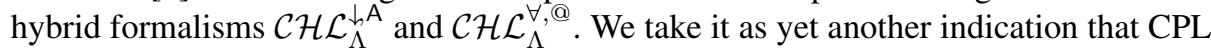
is natural and well-designed both as a generalization of FOL and "the" predicate relative of existing coalgebraic formalisms. Furthermore, due to a somewhat modal character of the CPL syntax, the correspondence is even closer and more natural than for ordinary FOL and additional results on the correspondence between sublanguages of CPL and various extensions of coalgebraic modal/hybrid logic become available. In the second part, we initiate the study of proof theory of CPL. We provide cut-free sequent systems for strongly one-step complete (S1SC) $\Lambda$-structures, which generalize the neighbourhood logic (and hence Chang's original setup). Our proof of cut-elimination is entirely syntactic and constructive.

\section{Syntax and Semantics}

Throughout the paper, we fix a modal similarity type $\Lambda$ consisting of modal operators with associated arities. We also fix a set $\Sigma$ of predicate symbols (with associated arities) and a set $\mathrm{V}^{i}$ of individual variables. The language $\mathcal{C P} \mathcal{L}_{\Lambda}$ of coalgebraic predicate logic is given by the grammar

$\mathcal{C P} \mathcal{L}_{\Lambda} \ni \phi, \psi::=x=y\left|P\left(x_{1}, \ldots, x_{n}\right)\right| \perp|\phi \rightarrow \psi| \forall x \phi \mid x \oslash\left\lceil x_{1}: \phi_{1}\right\rceil \ldots\left\lceil x_{n}: \phi_{n}\right\rceil$ 
where $\varnothing \in \Lambda$ is an $n$-ary modal operator, $P \in \Sigma$ is an $n$-ary predicate symbol and $x, y, x_{1}, \ldots, x_{n} \in \mathrm{V}^{i}$ are individual variables. For simplicity, we ignore function symbols which can be added in the same way as in [7].

In a formula $x \oslash\left\lceil x_{1}: \phi_{1}\right\rceil \ldots\left\lceil x_{n}: \phi_{n}\right\rceil, \varnothing$ is an $n$-ary modal operator, applied to $n$ arguments $\left\lceil x_{i}: \phi_{i}\right\rceil$, for $i=1, \ldots, n$. Here, $x_{i}$ is a comprehension variable. Given a first-order structure with carrier set $W$ and variable assignment $\vartheta,\lceil x: \phi\rceil$ denotes the set of all those states $w \in W$ such that $\phi$ holds under the modified assignment $\vartheta[x \mapsto w]$. Our informal reading of $\lceil x: \phi\rceil$ is 'the set of all $x$ such that $\phi$ '. As a consequence, the $n$-tuple $\left\lceil x_{1}: \phi_{1}\right\rceil \ldots\left\lceil x_{n}: \phi_{n}\right\rceil$ denotes an $n$-tuple of predicates on the carrier set, to which we can apply an $n$-ary modal operator $\varnothing$ in the usual way. The formula $x \oslash\left\lceil x_{1}: \phi_{1}\right\rceil \ldots\left\lceil x_{n}: \phi_{n}\right\rceil$ is then best understood as expressing that the property $\odot\left\lceil x_{1}: \phi_{1}\right\rceil \ldots\left\lceil x_{n}: \phi_{n}\right\rceil$ is true relative to the (interpretation of) $x$ in a firstorder structure.

Example 1. We have provided a number of examples of the use of CPL in a variety of situations already in [9] where consider CPL over probabilistic modal logic, over coalition logic and Pressburger modal logic. Here, we content ourselves with the following:

1. As originally noted by by Chang himself, coalgebraic predicate logic is particularly well-suited for reasoning about social situations and relationships between an individual and sets of individuals. Indeed, Chang's examples suggest an intensional reading of $\varnothing$ as 'useful' or 'enjoyable'. Given a unary modality $\square$ and a binary relation $S(x, y)$ that we read as ' $x$ speaks to $y$ ', the formula $x \square\lceil z: S(z, y)\rceil$ reads as ' $x$ finds it enjoyable to speak to $y$ ' where $x$ determines the truth of this sentence by inspecting the the set ' $\{z: S(z, y)\}$ ' of people speaking to $y$. The fact that whether or not $x$ finds it enjoyable to speak to $y$ may depend non-monotonically on the set of people $y$ converses with suggests to interpret $\square$ as a neighbourhood modality (as we will in fact do in Example 4).

2. Coalgebraic predicate logic can also be used to speak about 'losers', 'jerks' and 'politicians'. In [2], these terms are defined using hybrid logic over Kripke semantics where the underlying binary relation is understood as 'respects' or 'admires'. For example, a loser is understood as a person who lacks self-respect. In coalgebraic predicate logic, the fact that $x$ is a loser is expressed by the formula $x \square\lceil y: \neg(y=x)\rceil$. We read this formula as 'everybody whom $x$ respects has the property of being distinct from $x$ ', i.e. $x$ lacks self-respect. Accordingly, our interpretation of $\square$ (given in Detail in Example 4) in this example will be relational, and coincides with the Kripke-interpretation over relational models. We leave it to the reader to express their own (or [2]'s) notions of 'jerks' and 'politicians' in coalgebraic predicate logic. In Section 3, we will show that coalgebraic hybrid logic is in fact equi-expressive to coalgebraic predicate logic.

3. Coalgebraic predicate logic also extends, for instance, majority logic [11] to a first-order setting. If we take $x$ to be a politician if the majority of people known to them distrusts $x$, then the fact that $x$ is a politician is expressed by the formula $x \mathbf{M}\lceil y$ : $D(x, y)\rceil$ where $\mathbf{M}$ is the majority operator that we read 'the majority of' (and assume that majorities are taken among people that are known to an individual) and $D(x, y)$ is a 
binary relation that expresses that $x$ distrusts $y$. We will make this semantically precise, in the next example, by interpreting $\mathbf{M}$ as the majority operator of [11].

The semantics of coalgebraic predicate logic is given, as usual, in terms of a first order structure and a variable assignment. Crucially, the first-order structure must accommodate for the interpretation of the modalities present in the similarity type $\Lambda$ and must provide a uniform interpretation of modalities. We therefore understand first-order structures for $\Lambda$ as an enrichment of the standard notion of first-order structure with a device to interpret modalities. In our (coalgebraic) context, the interpretation of modal operators is given by $\Lambda$-structures.

Definition 2. A $\Lambda$-structure is an endofunctor $T:$ Set $\rightarrow$ Set on the category of sets, together with an assignment of predicate liftings, that is, a set-indexed family of maps

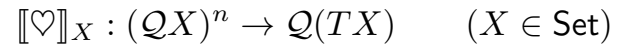

for every $n$-ary modal operator $\varnothing \in \Lambda$. Here $\mathcal{Q}$ is the contravariant powerset functor,

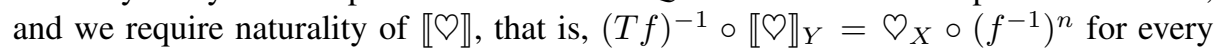
function $f: X \rightarrow Y$. We usually denote $\Lambda$-structures by the underlying endofunctor $T$, when the underlying assigned predicate liftings are clear.

In the remainder of this paper, we assume that our chosen set $\Lambda$ of modal operators comes equipped with a $\Lambda$-structure. We now take first-order structures to be $T$ coalgebras that are additionally equipped with an interpretation of the given relation symbols.

Definition 3. A first-order structure (for $\Lambda$ relative to a $\Lambda$-structure $T$ ) is a triple $\mathfrak{M}=$ $(C, \gamma, \pi)$ where $(C, \gamma)$ is a $T$-coalgebra, i.e. $C$ is a set and $\gamma: C \rightarrow T C$ a (transition) function, and $\pi$ is an interpretation of the predicate symbols, that is, $\pi(P) \subseteq C^{n}$ for all $n$-ary predicate symbols $P \in \Sigma$. We silenty identify $T$-coalgebras with first-order structures and leave the interpretation of predicate symbols implicit whenever this does not cause confusion.

The semantics of coalgebraic predicate logic is best explained as the algamation of coalgebraic modal logic and (standard) first-order logic. Given a $T$-coalgebra $(C, \gamma)$, formula $\phi$ of coalgebraic modal logic are interpreted as subsets $\llbracket \phi \rrbracket \subseteq C$. The crucial clause for modal operators is $\llbracket \oslash\left(\phi_{1}, \ldots, \phi_{n}\right) \rrbracket=\left\{c \in C \mid \gamma(c) \in \llbracket \oslash \rrbracket_{C}\left(\llbracket \phi_{1} \rrbracket, \ldots, \llbracket \phi_{n} \rrbracket\right)\right\}$, discussed in detail e.g. in [13]. Informally speaking, the (coalgebraic) interpretation of $\nabla\left(\phi_{1}, \ldots, \phi_{n}\right)$ is the set of individuals $c \in C$ that enjoy property $\varnothing$ (which depends on $\left.\phi_{1}, \ldots, \phi_{n}\right)$. In coalgebraic predicate logic, this interpretation is relativised to individuals: in a first-order structure $\mathfrak{M}=(C, \gamma, \pi)$, the formula $x \odot\left\lceil x_{1}: \phi_{1}\right\rceil \ldots\left\lceil x_{n}: \phi_{n}\right\rceil$ is true under a variable assigment $\vartheta$ if the individual $\vartheta(x)$ has property $\varnothing$ which now depends on the sets of individuals $x_{i}$ that have property $\phi_{i}\left(x_{i}\right)$.

Formally, we define truth $\mathfrak{M}, \vartheta \mid=\phi$ of a formula $\phi \in \mathcal{C P} \mathcal{L}_{\Lambda}$ in a first-order structure $\mathfrak{M}=(C, \gamma, \pi)$ relative to a variable assignment $\vartheta: \mathrm{V}^{i} \rightarrow C$ by the standard clauses for propositional and first-order connectives, augmented with

$$
\mathfrak{M}, \vartheta \models x \oslash\left\lceil x_{1}: \phi_{1}\right\rceil \ldots\left\lceil x_{n}: \phi_{n}\right\rceil \Longleftrightarrow \gamma(\vartheta(x)) \in \llbracket \oslash \rrbracket_{X}\left(\llbracket \phi_{1} \rrbracket_{\mathfrak{M}, \vartheta}^{x_{1}}, \ldots, \llbracket \phi_{n} \rrbracket_{\mathfrak{M}, \vartheta}^{x_{n}}\right)
$$


where $\llbracket \phi \rrbracket_{\mathfrak{M}, \vartheta}^{y}=\{c \in C \mid \mathfrak{M}, \vartheta[y \mapsto c] \models \phi\}$ (we usually drop the subscript $\mathfrak{M}, \vartheta$ ) and $\vartheta[y \mapsto c]$ is the same variable assignment as $\vartheta$ except it maps $y$ to $c$.

Example 4. We continue Example 1 and describe the structures that give rise to the interpretation put forward above.

1. Chang's original attempt generalises the neighbourhood interpretation of modal logics to the setting of full first-order logic. For a similarity type $\Lambda=\{\square\}$ containing a single unary operator, neighbourhood semantics is captured coalgebraically by the $\Lambda$-structure $\mathcal{N}=\mathcal{Q} \circ \mathcal{Q}$ together with the predicate lifting defined by

$$
\llbracket \square \rrbracket_{X}(A)=\{N \in \mathcal{N} X \mid A \in N\}
$$

which ensures that the standard modal neighbourhood semantics conincides with the coalgebraic semantics of modal formulae. In a first-order setting, this exhibits Chang's original language (and its interpretation) as a special case of coalgebraic predicate logic. In a first-order structure $(C, \gamma)$, every individual $c \in C$ induces a set $\gamma(c) \subseteq \mathcal{P}(C)$ of neighbourhoods such that - in the spirit of the example $-x$ finds it enjoyable to speak to $y$ if the set $\lceil z: S(z, y)\rceil$ is a (social) neighbourhood of $x$.

2. In [2], hybrid logic is used to define losers, jerks and politicians, where notions like respect or admiration are modelled by binary relations between individuals. We replace relations by $T$-coalgebras for $T C:=\mathcal{P} C(\mathcal{P}$ is the covariant powerset functor) and interpret the (unary) model operator $\square$ by $\llbracket \square \rrbracket_{C}(A):=\{B \in \mathcal{P} C \mid$ $B \subseteq A\}$ which gives the standard semantics. The formula $x \square\lceil y: \neg(y=x)\rceil$ then expresses that $x$ is a loser, i.e. lacks an arc along the relation expressing self-respect. We leave it to the reader to express the definitions of jerks and politicians given in [2]). Indeed, hybrid logic is translatable to the language discussed here, see below.

3. As a slight variation, we may consider a predicate version of majority logic [11] where we again co-algebraise the relational semantics. We interpret formulae involving an operator $\mathbf{M}$ (read 'the majority ...') over coalgebras of type $(C, \gamma$ : $C \rightarrow \mathcal{B} C$ ) where $\mathcal{B} C:=\{f: C \rightarrow \mathbb{N} \mid f(c) \neq 0$ only finitely often $\}$ using

$$
\llbracket \mathbf{M} \rrbracket_{C}(A):=\left\{f \in \mathcal{B} C \mid \sum_{x \in A} f(x)>\sum_{x \notin A} f(x)\right\} .
$$

This differs from the original semantics of op.cit. but induces the same set of true sentences. If we read $\mathbf{M}$ as the majority of people someone knows ... and $R(x, y)$ as likes, an unpopular person could be characterised by the sentence $x \mathbf{M}\{y$ : $\neg R(y, x)\}$ stipulating that the majority of people $x$ knows don't like $x$.

4. Frame classes can be combined: instead of using the relation symbol $R$ in the previous example, we could consider coalgebras $(C, \gamma: C \rightarrow T C)$ where $T C:=$ $\mathcal{B} C \times \mathcal{P} C$ gives a majority structure and a relational structure, and interpret the operators $\mathbf{M}$ and $\square$ by projecting out the components. Unpopular individuals are then characterised as satisfying $x \mathbf{M}\lceil y: y \square\lceil z: \neg(z=x)\rceil\rceil$. 


\section{Equipollence results}

\subsection{Coalgebraic Standard Translation for $\mathcal{C M} \mathcal{L}_{\Lambda}$}

The formulas $\mathcal{C M L}_{\Lambda}(\Sigma)$ of pure (coalgebraic) modal logic in the modal signature $\Lambda$ over $\Sigma$ (now all elements of $\Sigma$ are assumed to be of arity 1) are given by the grammar:

$$
\mathcal{C M}_{\mathcal{L}} \quad \phi, \psi::=P|\perp| \phi \rightarrow \psi \mid \varnothing\left(\phi_{1}, \ldots, \phi_{n}\right),
$$

where $P \in \Sigma$.

Satisfaction is defined with respect to $\mathfrak{M}=(C, \gamma, \pi)$ and a specific point $c \in C$ in a standard way, see e.g. $[14,15]$.

Proposition and Definition 5. Define the coalgebraic standard translation as

$$
\begin{aligned}
S T_{x}(P) & :=P(x), \\
S T_{x}\left(\varnothing\left(\phi_{1}, \ldots, \phi_{n}\right)\right) & :=x \oslash\left\lceil x: S T_{x}\left(\phi_{1}\right)\right\rceil \ldots\left\lceil x: S T_{x}\left(\phi_{n}\right)\right\rceil, \\
S T_{x}(\perp) & :=\perp \\
S T_{x}(\phi \rightarrow \psi) & :=S T_{x}(\phi) \rightarrow S T_{x}(\psi) .
\end{aligned}
$$

Then for any $\phi \in \mathcal{C} \mathcal{M} \mathcal{L}_{\Lambda}(\Sigma)$ and any $\mathfrak{M}=(C, \gamma, \pi), \vartheta$, c, we have $\mathfrak{M}, c \vDash \phi$ iff $\mathfrak{M}, \vartheta[x \mapsto c] \vDash S T_{x}(\phi)$.

This definition is more straightforward than the standard translation into FOL of modal logic over ordinary Kripke frames. Moreover, $S T_{x}$ uses only one variable from $\mathrm{V}^{i}$, namely $x$ itself. In fact, we can immediately observe that

Proposition 6. Whenever $\Sigma$ consists entirely of unary predicate symbols, the subset of $\phi \in \mathcal{C P} \mathcal{L}_{\Lambda}(\Sigma)$ obtained as the image of $S T_{x}$ for a fixed $x \in \mathrm{V}^{i}$ consists precisely of equality-free and quantifier-free formulae in the variable $x$.

Whereas the Van Benthem-Rosen theorem provided in [9] is a semantic characterization of $\mathcal{C} \mathcal{M} \mathcal{L}_{\Lambda}$ wrt $\mathcal{C P} \mathcal{L}_{\Lambda}$, Proposition 6 above is its syntactic counterpart. In fact, we can combine the two results to obtain

Corollary 7. Whenever $\Sigma$ consists entirely of unary predicate symbols (and there are no function symbols), the behaviourally-invariant (over finite structures) formulas of $\mathcal{C P} \mathcal{L}_{\Lambda}$ in one-free variable are up to equivalence (over finite structures) precisely the equality-free and quantifier-free formulas in the single-variable fragment of $\mathcal{C P} \mathcal{L}_{\Lambda}$.

No such syntactic characterization exists for formulas of ordinary first-order logic invariant under bisimulation. Of course, we can do better thanks to the somewhat more modal character of CPL syntax as compared to ordinary FOL.

\subsection{Hybrid Languages}

In this section, we generalize the results of [4]. Our ultimate goal is Theorem 13 below which establishes the equivalence of CPL with the hybrid languages $\mathcal{C H} \mathcal{L}_{\Lambda}^{\downarrow A}$ and 
$\mathcal{C H} \mathcal{L}_{\Lambda}^{\forall, @ ~}$. Both correspondences also hold for ordinary predicate logic over relational structures (FOL) and extend to CPL. We take this as yet another indication that CPL is natural and well-designed both as a generalization of FOL and "the" predicate logic cousin of existing coalgebraic formalisms.

This is our main, but not the only motivation. We progress towards this result step-bystep, extending the modal language gradually with new hybrid constructs. In this way, we reveal that a similar correspondence exists between natural fragments of CPL and weaker hybrid languages, most importantly between quantifier-free CPL and $\mathcal{C H} \mathcal{L}_{\Lambda}^{\downarrow @-}$ something which has no analogue in the FOL case.

Again, obviously the correspondence between fragments of CPL and extensions of CML is tighter than in the case of FOL and ML only due to the modal flavour of CPL. However, results such as Corollary 10 are useful spadework: any model-theoretic tool to be developed - say, a variant of E-F games - would be adequate for an extended coalgebraic modal formalism (e.g., $\mathcal{C H} \mathcal{L}_{\Lambda}^{\downarrow} @$ $)$ iff it is adequate for the corresponding fragment of CPL (e.g., the variable-free fragment), so we are free to work with whichever formalism we find more convenient at a given moment. This is closely related to our present research efforts. The straightforward correspondence also provides a good starting point for an extension of research programme sketched in [5] - see Remark 14 at the end of this section.

Given a supply of world variables $\mathrm{V}^{w}$ that we are going to keep fixed and implicit, we define the following coalgebraic hybrid languages

\begin{tabular}{ll}
\hline $\mathcal{C H} \mathcal{L}_{\Lambda}^{\downarrow @ @ ~}$ & $\phi, \psi::=z|P| \perp|\phi \rightarrow \psi| \oslash\left(\phi_{1}, \ldots, \phi_{n}\right)\left|@_{z} \phi\right| \downarrow z . \phi$ \\
$\mathcal{C H} \mathcal{L}_{\Lambda}^{\downarrow A}$ & $\phi, \psi::=z|P| \perp|\phi \rightarrow \psi| \varnothing\left(\phi_{1}, \ldots, \phi_{n}\right)|\mathrm{A} \phi| \downarrow z . \phi$ \\
$\mathcal{C H} \mathcal{L}_{\Lambda}^{\forall, @}$ & $\phi, \psi::=z|P| \perp|\phi \rightarrow \psi| \varnothing\left(\phi_{1}, \ldots, \phi_{n}\right)\left|@_{z} \phi\right| \forall z . \phi$ \\
\hline
\end{tabular}

where $z \in \mathrm{V}^{w}$. We refer the reader to, e.g, $[15,4,5]$ for the semantics. The extension of the standard translation to these formalism is unproblematic in some cases, just like in the case of ordinary hybrid logic over Kripke frames:

$$
S T_{x}(z):=x=z, \quad S T_{x}(\mathbf{A} \phi):=\forall x . S T_{x}(\phi), \quad S T_{x}(\forall z . \phi):=\forall z . S T_{x}(\phi) .
$$

One is tempted to put forward also

$$
S T_{x}\left(@_{z} \phi\right):=S T_{x}(\phi)[z / x], \quad S T_{x}(\downarrow z . \phi):=S T_{x}(\phi)[x / z] .
$$

However, with other clauses remaining the same, this could work only if $[z / x]$ denotes capture-avoiding substitution. Sadly, this in turn would entail forsaking the luxury of using just one designated variable for comprehension. Guillame Malod (see [6]) observed that if we restrict the supply of variables, a translation along the above linesindeed first proposed in the literature (which shows that the present discussion is less trivial than it might seem) — would fail even when embedding the hybrid logic over Kripke frames in the two-variable fragment of FOL. Malod's counterexample used nesting of modalities of level two, but as our translation uses just one designated variable, $S T$ would go wrong already on formulas of depth one. Just consider $S T_{x}(\downarrow z . \diamond z)$ : we would obtain $x \diamond\lceil x: x=x\rceil$, which is a formula with a completely different meaning. 
There are two ways out. First is to redefine

$$
\begin{aligned}
& \operatorname{STmod}_{x}\left(@_{z} \phi\right):=\forall x .\left(x=z \rightarrow S T_{x}(\phi)\right), \\
& \operatorname{STmod}_{x}(\downarrow z . \phi):=\forall z .\left(x=z \rightarrow S T_{x}(\phi)\right) .
\end{aligned}
$$

The second is to keep $S T$ for hybrid formulas as defined above and change the modal clause instead:

$$
S T_{x}\left(\varnothing\left(\phi_{1}, \ldots, \phi_{n}\right)\right):=x \oslash\left\lceil y: S T_{y}\left(\phi_{1}\right)\right\rceil \ldots\left\lceil y: S T_{y}\left(\phi_{n}\right)\right\rceil,
$$

where $y$ is the first (in some fixed enumeration) variable not used in $S T_{x}\left(\phi_{1}\right), \ldots, S T_{x}\left(\phi_{n}\right)$; by not used here we mean both free and bound usage. Furthermore, to ensure that the translation works correctly, we have to assume that neither $x$ nor $y$ appears in $\mathrm{V}^{w}$. While the requirement to use more bound variables can be cumbersome-particularly for infinite sets of formulas-we prefer this option, as it makes it easier to characterize weaker hybrid languages as suitable syntactic fragments of CPL.

We can now state a generalization of both Proposition 5 and corresponding results from the hybrid logic literature—see, e.g., [4] for references:

Proposition 8. For any $\phi \in \mathcal{C H} \mathcal{L}_{\Lambda}$ and any $\mathfrak{M}=(C, \gamma, \pi), \vartheta, c$, we have $\mathfrak{M}, \vartheta, c \vDash \phi$ iff $\mathfrak{M}, \vartheta[x \mapsto c] \vDash S T_{x}(\phi)$.

As is well-known in the hybrid logic community—see again [4] for references—-there is also a translation in the reverse direction for sufficiently expressive hybrid languages. This also generalizes to our setting, see Table 1 .

Table 1. Coalgebraic Hybrid Translation from quantifier-free CPL to $\mathcal{C H} \mathcal{L}_{\Lambda}^{\downarrow @}$

$$
\begin{array}{rlrl}
H T(P(x)) & :=@_{x} P & H T(x=y) & :=@_{x} y \\
H T(\perp) & :=\perp & H T(\phi \rightarrow \psi) & :=H T(\phi) \rightarrow H T(\psi) \\
H T\left(x \odot \left\lceily_{1}\right.\right. & \left.\left.: \phi_{1}\right\rceil \ldots\left\lceil y_{n}: \phi_{n}\right\rceil\right) & := & @_{x} \oslash\left(\downarrow y_{1} . H T\left(\phi_{1}\right), \ldots, \downarrow y_{n} . H T\left(\phi_{n}\right)\right)
\end{array}
$$

Proposition 9. For any $\phi \in \mathcal{C} \mathcal{P} \mathcal{L}_{\Lambda}$ and any $\mathfrak{M}=(C, \gamma, \pi), \vartheta$, we have

$$
\mathfrak{M}, \vartheta, c \vDash H T(\phi) \text { iff } \mathfrak{M}, \vartheta[x \mapsto c] \vDash \phi .
$$

Combining Propostions 9 and 8, we get:

Corollary 10. Whenever $\Sigma$ consists purely of unary predicates (and no function symbols), $\mathcal{C H} \mathcal{L}_{\Lambda}^{\downarrow} @$ is expressively equivalent to the quantifier-free fragment of $\mathcal{C} \mathcal{P} \mathcal{L}_{\Lambda}$, assuming $\mathrm{V}^{i}$ contains $\mathrm{V}^{w}$ plus a disjoint infinite supply of additional individual variables (used for comprehension).

Remark 11 (Quantifier-free CPL as the bounded fragment of FOL). In the case of ordinary FOL, the fragment equivalent to $\mathcal{C H} \mathcal{L}_{\Lambda}^{\downarrow}$ is characterized as the bounded fragment, see, e.g., [1]. In fact, our formula $x \odot\lceil y: \phi\rceil$, despite being quantifier-free on 
the surface, can be described as a form of bounded quantification. This can be formalized as a result stating that over coalgebras for the covariant powerset functor (Kripke frames), quantifier-free $\mathcal{C P} \mathcal{L}_{\Lambda}$ is equivalent to the bounded-fragment of ordinary FOL, where the role of $\bigcirc$ in $\mathcal{C P} \mathcal{L}_{\Lambda}$ is played by the binary relation symbol $R$ in FOL; details are left to the reader.

Remark 12 (Chang's original syntax). As already mentioned, our syntax is slightly different to the original one proposed by Chang [7]. In that paper, there were no explicit comprehension variables and even in the enriched syntax which allowed constants and function terms, the term on the left-hand side of $\odot$ had to be a variable. This variable was reused then on the right side of $\varnothing$ as the comprehension variable. In other words, Chang's $x \oslash \phi(x)$ was equivalent to ours $x \oslash\lceil x: \phi(x)\rceil$. In presence of quantifiers, which can be used to simulate the effect of capture-avoiding substitution as in STmod (this trick in fact stems back to Alfred Tarski), the two languages are obviously equivalent. But when considering fragments, as we do here, the equivalence breaks down; without quantifiers, Chang's syntax does not allow (2) and simple renaming of the comprehension variable on the right-hand side of $\odot$ as in (3) is not possible either.

There are two usual routes in hybrid logic to achieve full first-order expressivity. One is to add universal quantifiers over $\mathrm{V}^{w}$ in presence of the satisfaction operator @. The other is to add the global modality A in presence of the downarrow binder $\downarrow$ The hybrid translation is extended then as follows:

$$
\begin{aligned}
H T_{\forall @}(\forall x \cdot \phi) & :=\forall x \cdot H T(\phi) \\
H T_{\mathrm{A} \downarrow}(\forall x \cdot \phi) & :=\downarrow y \cdot \mathrm{A} \downarrow x \cdot \mathrm{A}(y \rightarrow \phi)
\end{aligned}
$$

In $H T_{\mathrm{A} \downarrow}$ we need the proviso that $y$ is not occurring in the whole formula.

Theorem 13. $\mathcal{C H} \mathcal{L}_{\Lambda}^{\downarrow \mathrm{A}}, \mathcal{C H} \mathcal{L}_{\Lambda}^{\forall, @ ~ a n d ~} \mathcal{C} \mathcal{P} \mathcal{L}_{\Lambda}$ are expressively equivalent.

As we can use $S \operatorname{Tmod}_{x}$ now and keep reusing $x$ as the comprehension variable, it is enough to assume that $\mathrm{V}^{i}=\mathrm{V}^{w} \cup\{x\}$. Since $@_{z} \phi$ is definable in presence of $\mathrm{A}$ (as $\mathrm{A}(z \rightarrow \phi), \downarrow$ is definable by the universal quantifier over $\mathrm{V}^{w}$ (as $\left.\forall z .(z \rightarrow \phi)\right)$ and $\mathrm{A}$ is definable by combination of $\forall$ and @ (as $\forall y$. @ ${ }_{y} \phi$, where $y$ is not used in $\phi$ ), we get in

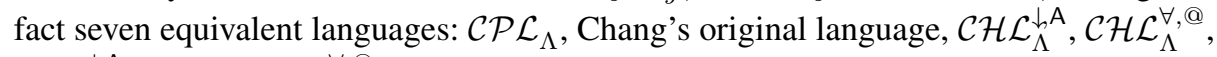
$\mathcal{C H} \mathcal{L}_{\Lambda}^{\downarrow A}$ with @, $\mathcal{C H} \mathcal{L}_{\Lambda}^{\forall, @ ~ w i t h ~} \downarrow$ and the jumbo hybrid language with all connectives introduced above.

Remark 14. The equivalences stated here extend to the case of $\mathcal{C H} \mathcal{L}_{\Lambda}$ and $\mathcal{C P} \mathcal{L}_{\Lambda}$ enriched with quantification over predicates (i.e., second-order languages). It would be interesting to follow more thoroughly the program of coalgebraic abstract model theory both above and below $\mathcal{C P} \mathcal{L}_{\Lambda}$ (see Ten Cate's PhD Thesis [5] for spadework in abstract model theory below first-order logic). 


\section{Proof theory}

\subsection{Axiomatisation of Coalgebraic Predicate Logic}

This paper's companion [9] already gives a complete Hilbert calculus for coalgebraic predicate logic that we review briefly here before giving an axiomatisation in terms of a cut-free sequent system. The crucial aspect of this system (and also of the sequent system that we will describe) are one-step rules that describe the geometry of the $\Lambda$ structure under consideration.

The modularity of coalgebraic predicate logic in the precise notion of structure necessitates that we cannot commit to a fixed set of rules. Instead, we import modal deduction rules into a first-order setting. It can be shown [13] that these deduction rules can be restricted to so-called one-step rules that have a very specific format. More precisely:

Definition 15. A $\left(\mathcal{C} \mathcal{M} \mathcal{L}_{\Lambda}\right)$ one-step rule over a similarity type $\Lambda$ has the form

$$
\frac{\Gamma_{1} \Rightarrow \Delta_{1} \quad \cdots \quad \Gamma_{k} \Rightarrow \Delta_{k}}{\bigcirc_{1} \boldsymbol{p}_{\mathbf{1}}, \ldots, \oslash_{n} \boldsymbol{p}_{\boldsymbol{n}} \Rightarrow \bigodot_{n+1} \boldsymbol{p}_{\boldsymbol{n}+\mathbf{1}}, \ldots, \bigcirc_{n+m} \boldsymbol{p}_{\boldsymbol{n}+\boldsymbol{m}}}(R)
$$

where $k, n, m \geq 0, \bigcirc_{1}, \ldots, \bigcirc_{n+m} \in \Lambda, \boldsymbol{p}_{\boldsymbol{i}}=\left(p_{i}^{1}, \ldots, p_{i}^{a(i)}\right)$ are vectors of propositional variables according to the arity $a(i)$ of $\varnothing_{i}$ and $\Gamma_{1}, \ldots, \Gamma_{k}, \Delta_{1}, \ldots, \Delta_{k} \subseteq\left\{p_{i}^{j} \mid\right.$ $1 \leq i \leq n, 1 \leq j \leq a(i)\}$. We denote the conclusion of $(R)$ by $\Gamma_{R} \Rightarrow \Delta_{R}$.

For the Hilbert-style axiomatisation of CPL, we write $\boldsymbol{x}, \boldsymbol{y}, \ldots$ for finite sequeneces of variables and define derivability $\mathcal{H R} \vdash$ as the the least set of formulae that is closed under modus ponens and contains axioms listed in Table 2. We have shown in [9] that

Table 2. Axioms of Coalgebraic Predicate Logic

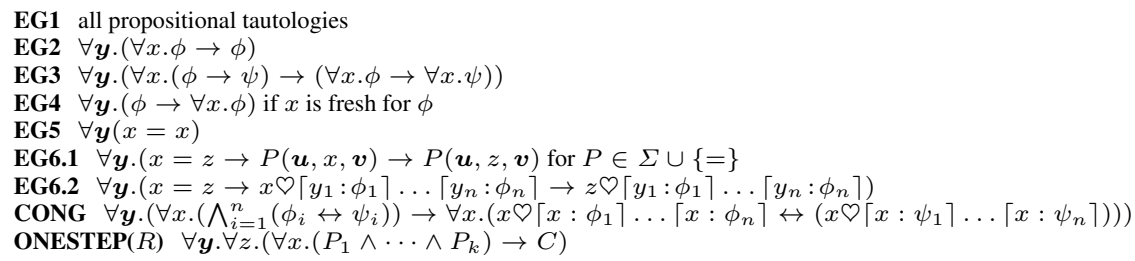

In ONESTEP, $R$ ranges over the one-step rules in $\mathcal{R}$ of the form above and $C=[\sigma, x, z]\left(\bigwedge \Gamma_{R} \rightarrow \bigvee \Delta_{R}\right)$ represents the conclusion of the rule and $P_{i}=\left(\bigwedge \Gamma_{i} \rightarrow \bigvee \Delta_{i}\right) \sigma$ its premises, where $\sigma$ sends each $p_{i}$ to a formula of $\mathcal{C} \mathcal{P} \mathcal{L}_{\Lambda}$ and $[\sigma, x, z]$ is the inductive extension of the map sending each $\bigodot_{i} \boldsymbol{p}_{\boldsymbol{i}}$ to $\left.z \bigodot_{i}\left\lceil x: \sigma\left(p_{i}^{1}\right)\right\rceil \cdots\left\lceil x: \sigma\left(p_{i}^{a(i)}\right)\right\rceil\right)$.

this calculus is complete for strongly one-step complete rule sets. One difference is that - as we are working here with (counterparts of) sequent-style rather than of Hilbertstyle one-step rules-our $\operatorname{ONESTEP}(R)$ has a slightly more general syntactic shape than the corresponding axiom in [9].

Definition 16. Given any supply of primitive symbols $D$ (which can be any set), define $\operatorname{Prop}(D)$ as the set of boolean combinations of $D$ and $\Lambda(D)=\left\{\odot\left(d_{1}, \ldots, d_{n}\right) \mid d_{1}, \ldots, d_{n} \in\right.$ 
$D$ and $\varnothing \in \Lambda$ is $n$-ary . For any $C \in$ Set, given a valuation $\tau: D \rightarrow \mathcal{P}(C)$, we write $C, \tau \models \alpha$ if $\tau(\alpha)=\top$ for all $\alpha \in \operatorname{Prop}(D)$. We understand $\llbracket \chi \rrbracket_{T C, \tau}$, i.e., the interpretation of $\chi \in \operatorname{Prop}(\Lambda(\operatorname{Prop}(D)))$ in the boolean algebra $\mathcal{P}(T C)$ under $\tau$, as the inductive extension of the assignment $\llbracket \curlyvee\left(\alpha_{1}, \ldots, \alpha_{n}\right) \rrbracket_{T X, \tau}=\llbracket \curlyvee \rrbracket_{C}\left(\tau\left(\alpha_{1}\right), \ldots, \tau\left(\alpha_{n}\right)\right)$. We write $T C, \tau=\chi$ if $\llbracket \chi \rrbracket_{T C, \tau}=T C$, and $t \models_{T C, \tau} \quad \chi$ if $t \in \llbracket \chi \rrbracket_{T C, \tau}$. A set $\Xi \subseteq \operatorname{Prop}(\Lambda(\operatorname{Prop}(D)))$ is one-step satisfiable with respect to $\tau$ if $\bigcap_{\chi \in \Xi} \llbracket \chi \rrbracket_{T C, \tau} \neq \emptyset$. If $D \subseteq \mathcal{P}(C)$ and $\tau$ is just the inclusion, we will usually drop it from the notation.

Definition 17. Let $\mathrm{P}$ be the set of propositional variables and $R=\Gamma_{1} \Rightarrow \Delta_{1}, \ldots, \Gamma_{k} \Rightarrow$ $\Delta_{k} / \Gamma_{R} \Rightarrow \Delta_{R}$ a one-step rule. We denote $\bigwedge\left\{\bigwedge \Gamma_{i} \rightarrow \bigvee \Delta_{i} \mid 1 \leq i \leq k\right\}(\in \operatorname{Prop}(\mathrm{P}))$ and $\bigwedge \Gamma_{R} \rightarrow \bigvee \Delta_{R}(\in \operatorname{Prop}(\Lambda(\mathrm{P})))$ by $\operatorname{Prem}(R)$ and $\operatorname{Conseq}(R)$, respectively. $\mathcal{R}$ is one-step sound if $T C, \tau \models \operatorname{Conseq}(R)$ whenever $C, \tau \models \operatorname{Prem}(R)$ for a valuation $\tau: \mathrm{P} \rightarrow \mathcal{P}(C)$, for all $R \in \mathcal{R}$. Given a set $\mathcal{R}$ of one-step rules and a valuation $\tau: \mathrm{P} \rightarrow \mathcal{P}(C)$, a set $\Xi \subseteq \operatorname{Prop}(\Lambda(\operatorname{Prop}(\mathrm{P})))$ is one-step consistent (with respect to $\tau)$ if the set $\Xi \cup\{\operatorname{Conseq}(R) \sigma \mid \sigma: \mathrm{P} \rightarrow \operatorname{Prop}(\mathrm{P}) ; R \in \mathcal{R} ; C, \tau \models \operatorname{Prem}(R) \sigma\}$ is propositionally consistent. A rule set $\mathcal{R}$ is strongly one-step complete (SISC) for a $\Lambda$ structure if for every set $C$, any $\Xi \subseteq \operatorname{Prop}(\Lambda(\operatorname{Prop}(\mathrm{P})))$ and any $\tau: \mathrm{P} \rightarrow \mathcal{P}(C), \Xi$ is one-step satisfiable with respect to $\tau$ whenever it is one-step consistent with respect to $\tau$. We say that a set of rules is finitary S1SC if the above holds whenever $\tau: \mathrm{P} \rightarrow \mathcal{P}_{\text {fin }}(C)$ (but not necessarily for arbitrary $\tau$ ).

The following was established in the companion paper [9].

Theorem 18. The Hilbert-calculus is sound and complete, i.e. $\mathcal{H R} \vdash \phi$ if and only if $\mathfrak{M}, \vartheta \models \phi$ for all first-order structures $\mathfrak{M}$ and all variable assignments $\vartheta$ provided $\mathcal{R}$ is strongly one-step complete (SISC) and one-step sound.

Strongly one-step complete rule sets are somewhat restrictive, but they do exist for Chang's original logic in terms of neighbourhood semantics and for coalition logic.

Here, we are complementing the axiomatisation of coalgebraic predicate logic by a cutfree, complete sequent calculus. Our basis is the system G3c of [17] that we extend with modal rules that describe the (fixed) $\Lambda$-structure $T$. We take sequents to be pairs $(\Gamma, \Delta)$, written $\Gamma \Rightarrow \Delta$ where $\Gamma, \Delta \subseteq \mathcal{C P} \mathcal{L}_{\Lambda}$ are finite multisets. The sequent calculus for coalgebraic predicate logic contains three types of rules: the standard logical rules for first-order logic, rules for equality and rules for the modal operators. The logical rules are standard as in Table 3. The formula introduced in the conclusion of a logical rule is called the principal formula of the rule.

The equality rules from Table 3 allow us to replace equals for equals both in predicate symbols and in modal formulae of the kind $x \oslash\left\lceil y_{1}: \phi_{1}\right\rceil \ldots\left\lceil y_{n}: \phi_{n}\right\rceil$. Equality rules do not have principal formulae.

To account for the modal operators, we incorporate the one-step rules $\mathcal{R}$ into the sequent system and write $\phi_{i}^{j}$ for $\sigma\left(p_{i}^{j}\right)$ as in $\operatorname{ONESTEP}(R)$. Then, we transform the axiom into its sequent form as follow:

$$
\frac{\left\{\left(\Gamma_{i} \sigma\right)[y / x] \Rightarrow\left(\Delta_{i} \sigma\right)[y / x] \mid 1 \leq i \leq k\right\} \quad y \text { fresh }}{z \bigcirc_{1}\left\lceil\boldsymbol{x}: \phi_{\mathbf{1}}\right\rceil, \ldots, z \bigcirc_{n}\left\lceil\boldsymbol{x}: \boldsymbol{\phi}_{\boldsymbol{n}}\right\rceil \Rightarrow z \bigcirc_{n+1}\left\lceil\boldsymbol{x}: \boldsymbol{\phi}_{\boldsymbol{n}+\mathbf{1}}\right\rceil, \ldots, z \bigcirc_{n+m}\left\lceil\boldsymbol{x}: \boldsymbol{\phi}_{\boldsymbol{n}+\boldsymbol{m}}\right\rceil}
$$

Furthermore, we repeat the information of the lower sequent in all the upper sequents to ensure the admissibility of contraction, and add a weakening context $\Sigma, \Theta$ to both 
Table 3. Sequent Rules of Coalgebraic Predicate Logic

Logical Rules

$$
\begin{aligned}
& (\mathrm{Ax}) \phi, \Gamma \Rightarrow \Delta, \phi \quad(\phi \text { atomic }) \quad(\mathrm{L} \perp) \perp, \Gamma \Rightarrow \Delta \\
& (\mathrm{L} \wedge) \frac{\phi, \psi, \Gamma \Rightarrow \Delta}{\phi \wedge \psi, \Gamma \Rightarrow \Delta} \quad(\mathrm{R} \wedge) \frac{\Gamma \Rightarrow \Delta, \phi \quad \Gamma \Rightarrow \Delta, \psi}{\Gamma \Rightarrow \Delta, \phi \wedge \psi}
\end{aligned}
$$

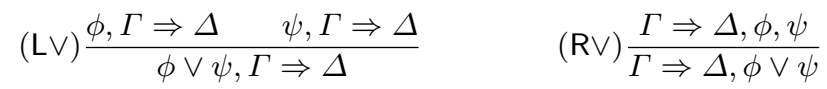

$$
\begin{aligned}
& (\mathrm{L} \rightarrow) \frac{\Gamma \Rightarrow \Delta, \phi \quad \psi, \Gamma \Rightarrow \Delta}{\phi \rightarrow \psi, \Gamma \Rightarrow \Delta} \quad(\mathrm{R} \rightarrow) \frac{\phi, \Gamma \Rightarrow \Delta, \psi}{\Gamma \Rightarrow \Delta, \phi \rightarrow \psi} \\
& \text { (L } \forall) \frac{\forall x \phi, \phi[y / x], \Gamma \Rightarrow \Delta}{\forall x \phi, \Gamma \Rightarrow \Delta} \quad(\mathrm{R} \forall) \frac{\Gamma \Rightarrow \Delta, \phi[y / x] y \text { fresh }}{\Gamma \Rightarrow \Delta, \forall x \phi}
\end{aligned}
$$

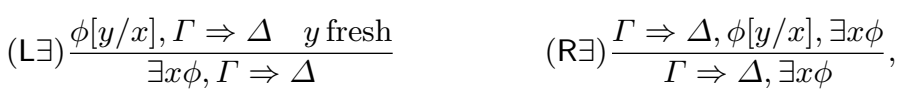

where $\phi[y / x]$ stands for the formula $\phi$ with all free occurrences of $x$ replaced by $y$ and the assumption of freshness means not occurring in the lower sequent of the rule.

Equality Rules

$$
(\operatorname{Ref}) \frac{x=x, \Gamma \Rightarrow \Delta}{\Gamma \Rightarrow \Delta} \quad \quad \text { (Repl) } \frac{\phi[y / z], x=y, \phi[x / z], \Gamma \Rightarrow \Delta}{x=y, \phi[x / z], \Gamma \Rightarrow \Delta}
$$

In $(\operatorname{Repl}), \phi$ is resticted to the atomic formulae.

$$
(\operatorname{Ren}) \frac{x=y, x \odot\left\lceil z: \phi_{1}\right\rceil \ldots\left\lceil z: \phi_{n}\right\rceil, y \curlyvee\left\lceil z: \phi_{1}\right\rceil \ldots\left\lceil z: \phi_{n}\right\rceil, \Gamma \Rightarrow \Delta}{x=y, x \odot\left\lceil z: \phi_{1}\right\rceil \ldots\left\lceil z: \phi_{n}\right\rceil, \Gamma \Rightarrow \Delta}
$$

Modal Rules $\mathcal{S}(\mathcal{R})$ : for every one-step rule of the form $R \in \mathcal{R}$,

$\mathcal{S}(R) \frac{\left\{\left(\Gamma_{i} \sigma\right)[y / x], \Gamma^{+} \Rightarrow \Delta^{+},\left(\Delta_{i} \sigma\right)[y / x] \mid 1 \leq i \leq k\right\} \quad y \text { fresh }}{\Sigma, z \bigcirc_{1}\left\lceil\boldsymbol{x}: \boldsymbol{\phi}_{\mathbf{1}}\right\rceil, \ldots, z \bigcirc_{n}\left\lceil\boldsymbol{x}: \boldsymbol{\phi}_{\boldsymbol{n}}\right\rceil \Rightarrow z \bigcirc_{n+1}\left\lceil\boldsymbol{x}: \boldsymbol{\phi}_{\boldsymbol{n}+\mathbf{1}}\right\rceil, \ldots, z \bigcirc_{n+m}\left\lceil\boldsymbol{x}: \boldsymbol{\phi}_{\boldsymbol{n}+\boldsymbol{m}}\right\rceil, \Theta}$

where $\sigma$ sends each $p_{i}^{j}$ to a formula $\phi_{i}^{j}$ of $\mathcal{C} \mathcal{P} \mathcal{L}_{\Lambda}$ and $\left\lceil\boldsymbol{x}: \phi_{\boldsymbol{i}}\right\rceil=\left\lceil x: \phi_{i}^{1}\right\rceil \ldots\left\lceil x: \phi_{i}^{a(i)}\right\rceil$ is a finite sequence of comprehension expressions according to the arity $a(i)$ of $\varnothing_{i}$ and $\Gamma^{+} \Rightarrow \Delta^{+}$ denotes the lower sequent.

(Admissible) Structual Rules

$$
\begin{gathered}
(\mathrm{W} L) \frac{\Gamma \Rightarrow \Delta}{\phi, \Gamma \Rightarrow \Delta} \quad(\mathrm{W} R) \frac{\Gamma \Rightarrow \Delta}{\Gamma \Rightarrow \Delta, \phi} \quad(\operatorname{Con} L) \frac{\Gamma, \phi, \phi \Rightarrow \Delta}{\Gamma, \phi \Rightarrow \Delta} \quad(\operatorname{Con} R) \frac{\Gamma \Rightarrow \Delta, \phi, \phi}{\Gamma \Rightarrow \Delta, \phi} \\
\text { (Cut) } \frac{\Gamma \Rightarrow \Delta, \phi \quad \phi, \Sigma \Rightarrow \Theta}{\Gamma, \Sigma \Rightarrow \Delta, \Theta}
\end{gathered}
$$


premise and conclusion. Finally, we obtain the desired form of $\mathcal{S}(R)$ in Table 3. The formulae $z \bigcirc_{i}\left\lceil\boldsymbol{x}: \phi_{\boldsymbol{i}}\right\rceil$ are called the principal formulae of $\mathcal{S}(R)$.

Example 19. If $(K)$ is the (one-step sound and one-step complete) rule set for the normal modal logic consisting of the rules

$$
\left(K_{n}\right) \frac{p \Rightarrow q_{1}, \ldots, q_{n}}{\diamond p \Rightarrow \diamond q_{1}, \ldots, \diamond q_{n}}
$$

for all $n \geq 0$, we obtain the following first-order version

$\mathcal{S}\left(K_{n}\right) \frac{\phi_{0}[y / z], \Sigma, z \diamond\left\lceil x: \phi_{0}\right\rceil \Rightarrow z \diamond\left\lceil x: \phi_{1}\right\rceil, \ldots, z \diamond\left\lceil x: \phi_{n}\right\rceil, \Theta, \phi_{1}[y / z], \ldots, \phi_{n}[y / z]}{\Sigma, z \diamond\left\lceil x: \phi_{0}\right\rceil \Rightarrow z \diamond\left\lceil x: \phi_{1}\right\rceil, \ldots, z \diamond\left\lceil x: \phi_{n}\right\rceil, \Theta}$

(where $y$ is fresh in the lower sequent) by the previous definition. Modal neighbourhood semantics is axiomatised by the one-step rule

$$
\text { (C) } \frac{p \Rightarrow q \quad q \Rightarrow p}{\square p \Rightarrow \square q}
$$

which expresses that $\square$ is a congruential operator. The first order version of (C) then reads

$$
\begin{aligned}
& \phi_{0}[y / x], \Sigma, z \square\left\lceil x: \phi_{0}\right\rceil \Rightarrow z \square\left\lceil x: \phi_{1}\right\rceil, \Theta, \phi_{1}[y / x] \\
& \phi_{1}[y / x], \Sigma, z \square\left\lceil x: \phi_{0}\right\rceil \Rightarrow z \square\left\lceil x: \phi_{1}\right\rceil, \Theta, \phi_{0}[y / x] \\
& \mathcal{S}(\mathrm{C}) \frac{\phi^{2}, z \square\left\lceil x: \phi_{0}\right\rceil}{\Rightarrow z \square\left\lceil x: \phi_{1}\right\rceil, \Theta}
\end{aligned}
$$

(where $y$ is fresh in the lower sequent) which we shall later see to provide a complete and cut-free axiomatisation of Chang's original logic.

If $\mathcal{R}$ is a set of one-step rules, we write $\mathcal{S R} \vdash \Gamma \Rightarrow \Delta$ if $\Gamma \Rightarrow \Delta$ can be derived using the logical and equality rules of Table 3 , together with the rules $\mathcal{S}(R)$ from Table 3 for every rule $R \in \mathcal{R}$. We write $\mathcal{S R C u t} \vdash \Gamma \Rightarrow \Delta$ if the cut rule (Cut) of Table 3 is used additionally. If $\mathfrak{M}=(C, \gamma, \pi)$ is a first-order structure over a $\Lambda$-structure $T$, we write $\mathfrak{M}, \vartheta \models \Gamma \Rightarrow \Delta$ if $\mathfrak{M}, \vartheta \mid \wedge \Gamma \rightarrow \bigvee \Delta$ and, as usual $\mathfrak{M} \models \Gamma \Rightarrow \Delta$ if $\mathfrak{M}, \vartheta \mid=$ $\Gamma \Rightarrow \Delta$ for all variable assignments $\vartheta$ and finally $T=\Gamma \Rightarrow \Delta$ if $\mathfrak{M} \models \Gamma \Rightarrow \Delta$ for all first-order structures $\mathfrak{M}$ over $T$.

We show soundness and completeness of the sequent system $\mathcal{S} \mathcal{R}$ by translating into, and from, the Hilbert system $\mathcal{H} \mathcal{R}$ which is known to be (semantically) complete. The translation - initially using (Cut) in the sequent system - relies on a few routine facts concerning structural rules that we now summarise (note that the definition of both $\mathcal{S R}$ and $\mathcal{S R C u t}$ does not involve structural rules). The structural rules are standard as in Table 3: we consider weakening both on the left and on the right and the rules of left and right contraction. Admissibility of weakening is standard:

Lemma 20. The rules of left weakening and right weakening are height-preserving admissible in $\mathcal{S R}$ and $\mathcal{S R C u t}$.

Proof. By induction on derivations. Note that weakening is built into modal rules $\mathcal{S}(R)$ that are derived from one-step rules in $\mathcal{R}$. The other cases than $\mathcal{S}(R)$ are done, e.g., as in [17, Lemma 3.5.3] and [10, Theorem 3.2.1 and Theorem 4.2.7]. 
Finally, we note one consequence of the congruence rule before we show that both systems $\mathcal{H} \mathcal{R}$ and $\mathcal{S} \mathcal{R}$ Cut have the same deductive power provided that the rules absorb congruence. We introduce the concept of absorption in a slightly more general form which will be used later.

Definition 21. We say that a set $S$ of sequents covers a set $S^{\prime}$ of sequents if each elements of $S^{\prime}$ is a subset of some element of $S^{\prime}$. We write $S \triangleright S^{\prime}$ if $S$ covers $S^{\prime}$ where we identify sequents with singleton sets. A set $\mathcal{R}$ of rules absorbs a rule $P / C$ if there exists a rule $R=Q / D \in \mathcal{R}$ such that $P \triangleright Q$ and $D \triangleright C$. A rule set absorbs congruence if it absorbs the rule

$$
(\text { Cong } \odot) \frac{p_{1} \Rightarrow q_{1} \quad \ldots \quad p_{n} \Rightarrow q_{n} \quad q_{1} \Rightarrow p_{1} \quad \ldots \quad q_{n} \Rightarrow p_{n}}{\curlyvee\left(p_{1}, \ldots, p_{n}\right) \Rightarrow \odot\left(q_{1}, \ldots, q_{n}\right)}
$$

and it absorbs monotonicity of $\odot$ in the $i$-th argument if the rule

$$
\left(\operatorname{Mon}_{i}\right) \frac{p_{i} \Rightarrow q_{i}}{\Gamma\left(p_{1}, \ldots, p_{n}\right) \Rightarrow \Upsilon\left(p_{1}, \ldots, p_{i-1}, q_{i}, p_{i+1}, \ldots p_{n}\right)}
$$

is absorbed.

Lemma 22. Suppose that $\mathcal{R}$ absorbs congruence. Then $\mathcal{S} \mathcal{R} \vdash \Gamma, \phi \Rightarrow \phi, \Delta$ for all formulas $\phi$.

Proof. Here we assume for simplicity that $\mathcal{R}$ consists of unary modal operators alone. As $\mathcal{R}$ absorbs congruence, the rule (Cong $\odot$ )

$$
\frac{\left\{\Gamma, \phi_{i}[y / x] \Rightarrow \psi_{i}[y / x], \Delta \mid 1 \leq i \leq n\right\} \quad\left\{\Gamma, \psi_{i}[y / x] \Rightarrow \phi_{i}[y / x], \Delta \mid 1 \leq i \leq n\right\}}{\Gamma, z \odot\lceil\boldsymbol{x}: \phi\rceil \Rightarrow z \odot\lceil\boldsymbol{x}: \boldsymbol{\psi}\rceil, \Delta}
$$

(where $y$ is fresh in the lower sequent and $n$ is the arity of $\varnothing$ ) is admissible in $\mathcal{S R}$ (and $\mathcal{S} \mathcal{R}$ Cut). This allows us to proceed by induction on the structure of $\phi$, where (Cong $\varnothing$ ) deals with the inductive case where $\phi$ is of the form $x \oslash\lceil y: \phi\rceil$.

One direction of the translation between the two proof systems can now be given as follows:

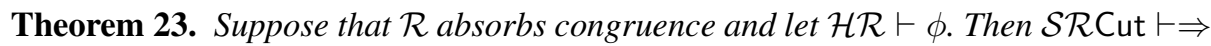
$\phi$.

Proof. First, we demonstrate admissibility of modus ponens in $\mathcal{S R C u t}$ by

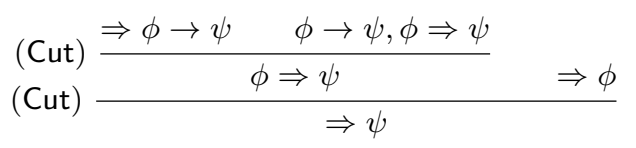

where the derivability of $\phi \rightarrow \psi, \phi \Rightarrow \psi$ is easily estabilished by Lemma 22. Note that, in our proof of the theorem, we need (Cut) only for this admissibility.

Then, it suffices to show that all the axioms of $\mathcal{H R}$ (recall Table 3 ) are derivable in $\mathcal{S R}$ (here we do not need the cut rule). Since this is easy to show for non-modal axioms, 
we focus on (EG6.2), (CONG) and $(\operatorname{ONESTEP}(R))$. First, (EG6.2) is derivable by (Ren) and Lemma 22. Second, the derivability of (CONG) follows from Lemma 22. Finally, let us move to the provability of $(\operatorname{ONESTEP}(R))$. Suppose that $R=\Gamma_{1} \Rightarrow$ $\Delta_{1}, \ldots, \Gamma_{k} \Rightarrow \Delta_{k} / \Gamma_{R} \Rightarrow \Delta_{R}$ is a one-step rule as in Definition 15 . We obtain the following derivation where $N=\{1, \ldots, n\}$ and $M=\{n+1, \ldots, n+m\}$ :

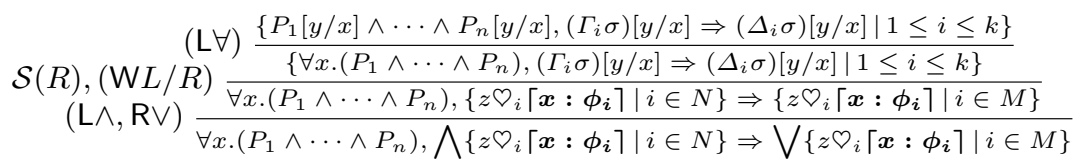

which shows derivability of the axiom $\operatorname{ONESTEP}(R)$ as the top sequent is readily seen to be provable in $\mathcal{S R}$ (recall that $P_{i}$ means $\left.\left(\bigwedge \Gamma_{i} \rightarrow \bigvee \Delta_{i}\right) \sigma\right)$.

For the converse direction, absorption of congruence is not required.

Theorem 24. Suppose that $\mathcal{S R C u t} \vdash \Gamma \Rightarrow \Delta$. Then $\mathcal{H} \mathcal{R} \vdash \wedge \Gamma \rightarrow \bigvee \Delta$.

Proof. It suffices to show that all the translations of the axioms and rules of $\mathcal{S R}$ are derivable in $\mathcal{H R}$. We can easily handle the cases of the axioms and rules for logical connectives of first-order logic. As for $\varnothing \in \Lambda$, the provability of the translation of $(\operatorname{Ren})$ and $\mathcal{S}(R)$ follows from (EG6.2) and (ONESTEP $(R))$, respectively.

As a corollary, we obtain (for the time being, in a calculus with cut) both soundness and completeness of the sequent calculus.

Corollary 25. Suppose that $\mathcal{R}$ is one-step sound and strongly one-step complete. Then $\mathcal{S R C u t} \vdash \Gamma \Rightarrow \Delta$ iff $\models \Gamma \Rightarrow \Delta$.

Proof. By Theorems 23 and 24 in conjunction with soundness and completeness of $\mathcal{H R}$ (Theorem 18). The absorption of congruence was shown in Proposition 5.12 of [12].

A paradigm example of a set of rules satisfying the assumptions of Corollary 25 is $C$ and its CPL translation $\mathcal{S}(\mathrm{C})$ from Example 19 above.

As we have remarked above, the assumption of strongly one-step complete rule sets is limiting in that there are only few examples. The companion paper [9] gives a complete Hilbert-style axiomatisation also for bounded operators. We repeat the definition for convenience:

Definition 26. A modal operator $\odot$ is $k$-bounded in $i$-th argument for $k \in \mathbb{N}$ and with respect to a $\Lambda$-structure $T$ if for every $C \in$ Set and every $A_{1}, \ldots, A_{n} \subseteq C$,

$$
\llbracket \oslash \rrbracket_{C}\left(A_{1}, \ldots, A_{n}\right)=\bigcup_{B \subseteq A_{i}, \# B \leq k} \llbracket \oslash \rrbracket_{C}\left(A_{1}, \ldots, A_{i-1}, B, A_{i+1}, \ldots, A_{n}\right) .
$$

Note that this implies in particular that $\varnothing$ is monotonic in the $i$-th argument. Examples of bounded modalities include the standard $\diamond$ of relational modal logic interpreted 
over Kripke frames, graded modalities over multigraphs and we refer to [15] for more examples. In the Hilbert-calculus, boundedness is reflected syntactically by the axiom

$$
\begin{aligned}
& \operatorname{BDPL}_{k}, i \forall \bar{y} .\left(x \oslash\left\lceil y_{1}: \phi_{1}\right\rceil \ldots\left\lceil y_{n}: \phi_{n}\right\rceil \leftrightarrow \exists z_{1} \ldots z_{k} .\left(x \oslash\left\lceil y_{1}: \phi_{1}\right\rceil \ldots\left\lceil y_{i-1}: \phi_{i-1}\right\rceil\right.\right. \\
&\left.\left.\left\lceil y_{i}: y_{i}=z_{1} \vee \cdots \vee y_{i}=z_{k}\right\rceil\left\lceil y_{i+1}: \phi_{i+1}\right\rceil \ldots\left\lceil y_{n}: \phi_{n}\right\rceil \wedge \bigwedge_{j \leq k} \phi_{i}\left[z_{j} / y_{i}\right]\right)\right)
\end{aligned}
$$

where each $z_{i}$ is fresh for all the $y_{i} \mathrm{~s}$ and $\phi_{i} \mathrm{~s}$. The derivability predicate induced by exteding the Hilbert calculus $\mathcal{H} \mathcal{R}$ by the boundedness axiom above gives completeness under weaker conditions.

Definition 27. We write $\mathcal{B H} \mathcal{R} \vdash \phi$ if $\phi$ is derivable in $\mathcal{H} \mathcal{R}$ where additionally $\left(\mathrm{BDPL}_{k, i}\right)$ is used for every operator that is $k$-bounded in the $i$-th argument.

Strictly speaking, the derivability predicate $\mathcal{B H} \mathcal{R}$ should include information about precisely which operators are assumed to be $k$-bounded in the $i$-th argument, but this will always be clear from the context. In the presence of boundedness, completeness of the Hilbert-calculus can be established under weaker conditions, where we again refer to [9] for details:

Theorem 28. Suppose that $\mathcal{R}$ is one-step sound and finitary strongly one-step complete over a $\Lambda$-structure T. Let each operator be bounded in every argument. Then $\mathcal{B H} \mathcal{R} \vdash \phi$ iff $\mathfrak{M}, \vartheta \models \phi$ for every first-order structure $\mathfrak{M}$ and every variable assignment $\vartheta$.

We can reflect boundedness in the sequent calculus by adding a paste rule, similar in spirit to the paste rule of hybrid logic [3, Section 7] which was generalised to a coalgebraic setting in [15]. In a sequent setting, this rule takes the form

$$
\begin{gathered}
\Gamma, x \oslash\left\lceil x_{1}: \phi_{1}\right\rceil \cdots\left\lceil x_{i-1}: \phi_{i-1}\right\rceil\left\lceil y: \bigvee_{1 \leq j \leq k} y=z_{j}\right\rceil\left\lceil x_{i+1}: \phi_{i+1}\right\rceil \cdots\left\lceil x_{n}: \phi_{n}\right\rceil, \\
\left(\operatorname{Paste}_{i}^{k}\right) \frac{\phi\left[z_{1} / y\right], \ldots, \phi\left[z_{k} / y\right] \Rightarrow \Delta \quad z_{1}, \ldots, z_{k} \text { fresh }}{\Gamma, x \oslash\left\lceil x_{1}: \phi_{1}\right\rceil \cdots\left\lceil x_{i-1}: \phi_{i-1}\right\rceil\lceil y: \phi\rceil\left\lceil x_{i+1}: \phi_{i+1}\right\rceil \cdots\left\lceil x_{n}: \phi_{n}\right\rceil \Rightarrow \Delta},
\end{gathered}
$$

where $z_{1}, \ldots, z_{k}$ are pairwise distinct fresh variables. Additional use of the above pasterule in the system $\mathcal{S R}$ is denoted by $\mathcal{B S R}$, that is, we write $\mathcal{B S R} \vdash \Gamma \Rightarrow \Delta$ if $\Gamma \Rightarrow \Delta$ is derivable in $\mathcal{S} \mathcal{R}$ where $\left(\operatorname{Paste}_{i}^{k}\right)$ may additionally be applied for every modality that is $k$-bounded in the $i$-th argument.

In what follows, we assume that $\mathcal{R}$ absorbs the congruence rules for all $\varnothing \in \Lambda$ and monotonicity of all operators that are $k$-bounded in the $i$-th argrument.

Lemma 29. Suppose that $\mathcal{R}$ absorbs congruence. Then, the replacement axiom $x=$ $y, \phi[x / z] \Rightarrow \phi[y / z]$ is derivable in $\mathcal{S R}$.

Proof. By induction on $\phi$ (note that we do not need the cut rule in this proof). It suffices to check the case where $\phi$ is of the form $v \oslash\lceil\boldsymbol{w}: \boldsymbol{\psi}\rceil$, since the other cases than $z \bigcirc\lceil\boldsymbol{x}: \boldsymbol{\psi}\rceil$ are done, e.g., as in [17, Lemma 4.7.2 (i)] and [10, Lemma 6.5.2]. For simplicity, let us consider the case where $\phi$ is $v \oslash\lceil w: \psi\rceil$ (i.e., $\varnothing$ is unary). In order to 
show the derivability of $x=y,(v \odot\lceil w: \psi\rceil)[x / z] \Rightarrow(v \odot\lceil w: \psi\rceil)[y / z]$, here we only consider a case where $z \equiv v$ and $z \not \equiv w$ as follows.

$$
\begin{aligned}
& \frac{y=x, \psi[y / z]\left[w^{\prime} / w\right] \Rightarrow \psi[x / z]\left[w^{\prime} / w\right]}{\frac{x=x, x=y, \psi[y / z]\left[w^{\prime} / w\right] \Rightarrow \psi[x / z]\left[w^{\prime} / w\right]}{x}}(\operatorname{Repl}),(\mathbf{W} L)
\end{aligned}
$$

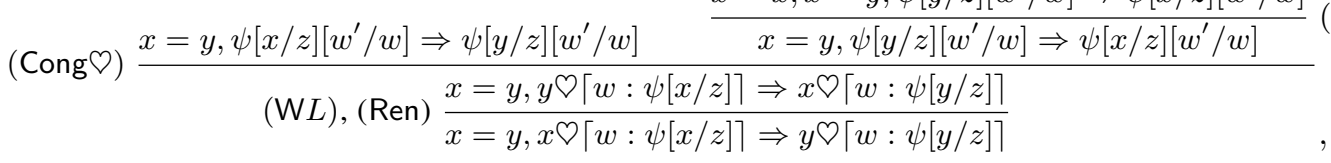

where $w^{\prime}$ is fresh in the lower sequent of $\operatorname{Cong}(\mathcal{Q})$ and two top sequents are provable in $\mathcal{S R}$ by induction hypothesis.

Theorem 30. Suppose that $\mathcal{R}$ absorbs congruence and monotonicity in the $i$-th argument of every operator that is $k$-bounded in the $i$-th argument. Then $\mathcal{B H} \mathcal{R} \vdash \phi$ implies that $\mathcal{B S R C u t} \vdash \Rightarrow \phi$.

Proof. First of all, if $\mathcal{R}$ absorbs monotonicity in the $i$-th argument of $\varnothing \in \Lambda$, the rule

$$
\left(\operatorname{Mon}_{i}\right) \frac{\Gamma, \phi_{i}[y / x] \Rightarrow \psi[y / x], \Delta}{\Gamma, z \bigcirc\lceil x: \phi\rceil \Rightarrow z ९\left\lceil x: \phi_{1}\right\rceil \ldots\left\lceil x: \phi_{i-1}\right\rceil\lceil x: \psi\rceil\left\lceil x: \phi_{i+1}\right\rceil \ldots\left\lceil x: \phi_{n}\right\rceil, \Delta}
$$

(where $y$ is fresh in the lower sequent) is admissible in $\mathcal{B S R}$ (and $\mathcal{B S R}$ Cut). Almost all the arguments are the same as the proof of Theorem 23, except that we need to show the provablity of (BDLP) by (Paste) (The only place we need the cut rule is the derivability of modus ponens). More precisely, we can show the left-to-right implication of (BDPL) by means of $\left(\mathrm{Paste}_{i}^{k}\right)$ and $\left(\mathrm{Mon}_{i}\right)$ gives the reverse direction. For example, when $\odot$ is unary and 1-bounded, the derivability of the right-to-left direction of (BDPL) is demonstrated as follows.

$$
\begin{aligned}
& \begin{array}{c}
(\mathrm{W} L) \frac{w=v, \phi[w / y] \Rightarrow \phi[v / y]}{v=v, v=w, w=v, \phi[w / y] \Rightarrow \phi[v / y]} \\
(\operatorname{Repl}) \frac{v=v, v=w, \phi[w / y] \Rightarrow \phi[v / y]}{v=}
\end{array}
\end{aligned}
$$

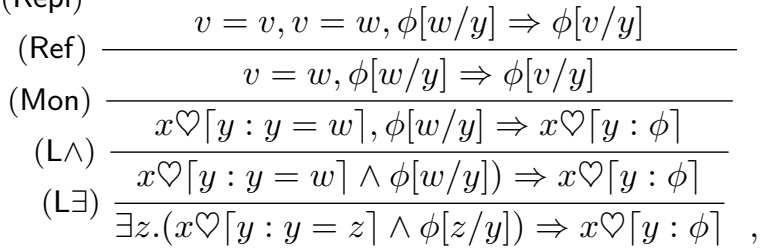

where the top sequent is the replacement axiom, which is derivable by Lemma 29.

The reverse direction of Theorem 30 is established analogously to Theorem 24 and again absorption properties are not needed.

Theorem 31. $\mathcal{B S R C u t} \vdash \Gamma \Rightarrow \Delta$ only if $\mathcal{B H \mathcal { R }} \vdash \wedge \Gamma \rightarrow \bigvee \Delta$

Proof. The only difference from the proof of Theorem 23 is to need to care about the translation of Paste. However, we can easily establish this by the axiom (BDLP).

As in the non-bounded case we obtain semantic soundness and completeness, but under weaker coherence conditions. 
Corollary 32. Suppose that $\mathcal{R}$ is one-step sound and strongly finitary one-step complete. Then $\mathcal{B S R C u t} \vdash \Gamma \Rightarrow \Delta$ iff $\models \Gamma \Rightarrow \Delta$.

Proof. By Theorems 30 and 31. Note that absorption of congruence and monotonicity follows from (strong, finitary) one-step completeness as in Proposition 5.12 of [12].

A canonical example of a rule set satisfying the assumptions of the above corollary can be obtained by taking $\mathrm{K}$ of Example 19 and extending it with $\left(\operatorname{Paste}_{i}^{k}\right)$ for $i=k=n=$ 1 .

\subsection{Elimination of Contraction and Cut}

Note that a priori we cannot expect that cut elimination holds for cuts between two instances of modal rules: the set $\mathcal{R}$ of one-step rules can possibly consist of a single rule, and a cut between this rule and itself may not be derivable. We therefore need to impose an additional requirement, cut and contraction closure to deal with this case.

Definition 33. Let $S$ be a finite set of sequents. The set of sequents that can be derived from premises $S$ using (only) the contraction rules is denoted by $\operatorname{Con}(S)$. Similarly, the set of all sequents that can be derived from premises in $S$ using (only) the cut rule is denoted by $\operatorname{Cut}(S)$. A rule set $\mathcal{R}$ absorbs contraction if, for all rules $R=P / C \in \mathcal{R}$ and all $C^{\prime} \in \operatorname{Con}(C)$ there exists a rule $R^{\prime}=Q / D \in \mathcal{R}$ such that $\operatorname{Con}(P) \triangleright Q$ and $D \triangleright C^{\prime}$. A rule set $\mathcal{R}$ absorbs cut, if for all pairs of rules in $\mathcal{R}$

$$
\left(R_{1}\right) \frac{P_{1}}{\Gamma_{1} \Rightarrow \Delta_{1}, \phi} \quad\left(R_{2}\right) \frac{P_{2}}{\phi, \Gamma_{2} \Rightarrow \Delta_{2}}
$$

there is a rule $R=P / C \in \mathcal{R}$ such that $\operatorname{Cut}\left(P_{1} \cup P_{2}\right) \triangleright P$ and $C \triangleright \Gamma_{1}, \Gamma_{2} \Rightarrow \Delta_{1}, \Delta_{2}$.

Informally, absorption of cut and contraction allows us to replace an application of cut or contraction to the conclusions of rules in $\mathcal{R}$ by a possibly different rule with possibly weaker premises and stronger conclusion. While these definitions are purely syntactic, a semantic characterisation has been given in [12] in terms of one-step cut-free completeness. For many $\Lambda$-structures, including those for probabilistic and graded modal logic, the modal logic $K$, the logic of (monotone) neighbourhood frames, one-step cutfree complete rule sets are known. In particular, these rule sets satisfy absorption of cut, contraction and congruence [12, Section 5].

The absorption requirements directly translate into proof-theoretic properties of the associated sequent calculus for coalgebraic predicate logic that we now collect. Note that weakening is built into one-step rules so that weakening is always admissible without further assumptions.

Lemma 34. All the logical and equality rules of $\mathcal{S} \mathcal{R}$ are height-preserving invertible.

Lemma 35. If $\mathcal{S R} \vdash \Gamma \Rightarrow \Delta$ and $y$ is fresh in $\Gamma$ and $\Delta$, then If $\mathcal{S R} \vdash \Gamma[y / x] \Rightarrow$ $\Delta[y / x]$ with the same height of derivation.

Proposition 36. Suppose $\mathcal{R}$ is a set of one-step rules over a similarity type $\Lambda$. If $\mathcal{R}$ absorbs contraction, then the rules $(\operatorname{Con} L)$ and $(\operatorname{Con} R)$ are admissible in $\mathcal{S R}$. 
Proof. By induction on the structure of proofs with the help of Lemmas 34 and 35. We have to use absorption of contraction to replace an application of a contraction rule to the conclusion of $\mathcal{S}(R)$ in order to replace the application of $\mathcal{S}(R)$ by (a possibly different) rule $\mathcal{S}\left(R^{\prime}\right)$.

We now turn to cut-elimination, where the majority of the cases are straightforward, and in fact identical to the cut-elimination proof in first-order logic.

Theorem 37 (Cut Elimination). Suppose that $\mathcal{R}$ absorbs cut and contraction. Then the cut rule is admissible in $\mathcal{S R}$.

Proof. We proceed by double induction on the size of the cut formula and the size of the proof tree. In all cases that do not involve the application of a rule $\mathcal{S}(R)$ for some $R \in \mathcal{R}$ it is straightforward to either propagate the cut upwards or to replace the cut by a smaller cut formula using the fact that contraction is admissible. Now fix a one-step rule $R$ and consider the cuts involving $\mathcal{S}(R)$. For cuts between $\mathcal{S}(R)$ and another $\mathcal{S}\left(R^{\prime}\right)$, the cut may be eliminated by the fact that $\mathcal{R}$ absorbs cut. For cuts between $\mathcal{S}(R)$ and an equality rule, the cut can be propagated upwards in the proof tree. For a cut between $\mathcal{S}(R)$ and a logical rule, we distinguish two cases depending on whether or not the cut formula $A$ is principal in one of the rules.

In case $A$ not principal in $\mathcal{S}(R)$ the cut may be eliminated by choosing a different weakening context in the application of $\mathcal{S}(R)$. In case $A$ is principal in $\mathcal{S}(R)$ we observe that $A$ is of the form $x \oslash\lceil y: \phi\rceil$ and therefore cannot be principal in a logical rule. This allows us to propagate the cut upwards in the proof tree.

As an immediate corollary, we obtain completeness of the cut-free calculus assuming that $\mathcal{R}$ is strongly one-step complete:

Corollary 38. Suppose that $\mathcal{R}$ is one-step sound and strongly one-step complete over a $\Lambda$-structure T. Then $=\Gamma \Rightarrow \Delta$ iff $\mathcal{S R} \vdash \Gamma \Rightarrow \Delta$.

Proof. This follows from Theorem 37 with the help of Proposition 5.11 and 5.12 of [12], the latter asserting precisely the absorption of cut and congruence.

The situation is more complex in presence of bounded operators where completeness of the Hilbert calculus is only guaranteed in presence of (BDPL), and completeness of the associated sequent calculus relies on $\left(\mathrm{Paste}_{i}^{k}\right)$. The difficulty in a proof of cutelimnation is a cut-end derivation where a cut is performed on $x \odot\left\lceil y_{1}: \phi_{1}\right\rceil \ldots\left\lceil y_{n}\right.$ : $\left.\phi_{n}\right\rceil$ which is introduced by $\left(\operatorname{Paste}_{i}^{k}\right)$ and a (one-step) rule where the same formula is principal. We leave this as an open problem:

Open Problem 39. Is there a way to modify the rules of $\mathcal{B S R}$ so that completeness with respect to $\mathcal{B H} \mathcal{R}$ holds and cut is admissible?

\section{Conclusions}

We believe that results obtained here, particularly in Section 3 strengthens the claims first made in [9] concerning naturality of CPL as a (or perhaps "the"?) predicate counterpart of existing coalgebraic formalisms. As concerns sequent systems and cut-elimination 
results in Section 4, we have fully achieved our goals for those functors and signatures which are "sufficiently neighbourhood-like" (S1SC). We are presently working on the intriguing question whether a constructive proof of cut-elimination can be given on the "Kripke-like" end, i.e., Open Problem 39. We refer the reader to [9] for more on open problems and future work.

\section{References}

1. Carlos Areces and Balder ten Cate. Hybrid logics. In P. Blackburn, J. van Benthem, and F. Wolter, editors, Handbook of Modal Logic. Elsevier, 2007.

2. Patrick Blackburn. Representation, reasoning, and relational structures: a hybrid logic manifesto. Logic Journal of the IGPL, 8(3), 2000.

3. Patrick Blackburn, Maarten de Rijke, and Yde Venema. Modal Logic. Cambridge University Press, 2001.

4. Patrick Blackburn and Balder ten Cate. Pure extensions, proof rules, and hybrid axiomatics. Studia Logica, 84(2):277-322, 2006.

5. Balder ten Cate. Model theory for extended modal languages. $\mathrm{PhD}$ thesis, University of Amsterdam, 2005. ILLC Dissertation Series DS-2005-01.

6. Balder ten Cate and Massimo Franceschet. On the complexity of hybrid logics with binders. In C.-H. Luke Ong, editor, Proc. CSL 2005, volume 3634 of Lecture Notes in Computer Science, pages 339-354. Springer, 2005.

7. C. Chang. Modal model theory. In A. Mathias and H. Rogers, editors, Cambridge Summer School in Mathematical Logic, volume 337 of Lecture Notes in Mathematics, pages 599-617. Springer, 1973.

8. Helle Hvid Hansen, Clemens Kupke, and Eric Pacuit. Neighbourhood structures: Bisimilarity and basic model theory. Log. Methods Comput. Sci., 5, 2009.

9. Tadeusz Litak, Dirk Pattinson, Katsuhiko Sano, and Lutz Schröder. Coalgebraic predicate logic. In A. Czumaj et al., editor, Proceedings of the 39th International Colloquium on Automata, Languages and Programming (ICALP) 2012, Part II, volume 7392 of LNCS, pages 299-311. Springer, Heidelberg, 2012.

10. S. Negri and J. Von Plato. Structural Proof Theory. Cambridge University Press, 2001.

11. E. Pacuit and S. Salame. Majority logic. In D. Dubois, C. Welty, and M.-A. Williams, editors, Proc. KR 2004, pages 598-605. AAAI Press, 2004.

12. D. Pattinson and L. Schröder. Cut elimination in coalgebraic logics. Information and Computation, 208:1447-1468, 2010.

13. Lutz Schröder. A finite model construction for coalgebraic modal logic. J. Log. Algebr. Prog., 73:97-110, 2007.

14. Lutz Schröder and Dirk Pattinson. Coalgebraic correspondence theory. In Foundations of Software Science and Computations Structures, FOSSACS 2010, volume 6014 of LNCS, pages 328-342. Springer, 2010.

15. Lutz Schröder and Dirk Pattinson. Named models in coalgebraic hybrid logic. In JeanYves Marion and Thomas Schwentick, editors, STACS, volume 5 of LIPIcs, pages 645-656. Schloss Dagstuhl - Leibniz-Zentrum fuer Informatik, 2010.

16. Joseph Sgro. The interior operator logic and product topologies. Trans. AMS, 258(1):pp. 99-112, 1980.

17. A. Troelstra and H. Schwichtenberg. Basic Proof Theory. Cambridge University Press, 1996. 